\title{
Clifford algebra-based structure filtering analysis for geophysical vector fields
}

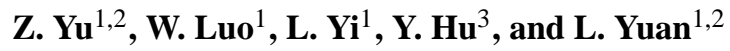 \\ ${ }^{1}$ Key Laboratory of Virtual Geographic Environment, Ministry of Education, Nanjing Normal University, \\ No.1 Wenyuan Road, Nanjing, China \\ ${ }^{2}$ Jiangsu Provincial Key Laboratory for Numerical Simulation of Large Scale Complex Systems, Nanjing Normal University, \\ No.1 Wenyuan Road, Nanjing, China \\ ${ }^{3}$ Department of Computer Science and Technology, Nanjing Normal University, No.1 Wenyuan Road, Nanjing, China
}

Correspondence to: L. Yuan (yuanlinwang@ @ nnu.edu.cn)

Received: 28 May 2013 - Revised: 24 June 2013 - Accepted: 25 June 2013 - Published: 31 July 2013

\begin{abstract}
A new Clifford algebra-based vector field filtering method, which combines amplitude similarity and direction difference synchronously, is proposed. Firstly, a modified correlation product is defined by combining the amplitude similarity and direction difference. Then, a structure filtering algorithm is constructed based on the modified correlation product. With custom template and thresholds applied to the modulus and directional fields independently, our approach can reveal not only the modulus similarities but also the classification of the angular distribution. Experiments on exploring the tempo-spatial evolution of the 2002-2003 El Niño from the global wind data field are used to test the algorithm. The results suggest that both the modulus similarity and directional information given by our approach can reveal the different stages and dominate factors of the process of the El Niño evolution. Additional information such as the directional stability of the El Niño can also be extracted. All the above suggest our method can provide a new powerful and applicable tool for geophysical vector field analysis.
\end{abstract}

\section{Introduction}

The vector field data not only have higher data dimensions, but also show complex structures and express more attractive, meaningful and vivid information (Mendoza et al., 2010). Traditional vector field analysis methods based on vector algebra and calculus are mostly used for ideal fields. They are sensitive to the noise of data and their robustness need further tests (Tafti and Michael, 2011). Statistical methods such as EOF/PCA (Empirical Orthogonal Function/ Principal Components Analysis) divide the vector field signal into two or more scalar signals, which will lead to the segmentary, ambiguous or even wrong modes (Paulus and Mars, 2006; Yu et al., 2011). The development of pattern filtering method for feature extraction may be helpful for the processing and analysis of vector field data (Yuan et al., 2013).

Template matching is one of the most commonly used technologies for feature filtering. Clifford convolution is applied to compute the direction and similarities between vector field and ideal vector field template (Ebling and Scheuermann, 2005), and it is also used in trajectory and eddy characteristics researches (Brassington et al., 2011). These studies provide references for developing more powerful vector field filtering technologies. Aiming at filtering meaningful geophysical signals, the new-developed method should meet the following criteria: (1) it should be noise-insensitive, and can directly support complicated geological observation data as the template; (2) both the amplitude similarities and directions should be carefully integrated in the filtering process; (3) it should allow a threshold range of both amplitude similarities and angular difference; (4) it should have efficient performance, and can support global scale analysis.

In this article, the Clifford convolution is introduced as the foundation to construct the vector field data filtering algorithm. The vector field data are expressed by the Clifford algebra bases and split into the modulus fields and unit length direction fields to separate the amplitude and direction information. The Clifford convolution is applied into the original vector field and the template data. The scalar part and the 
angular part of the convolution result are discussed. Then, both the scalar and the angular part of the convolution are replaced with the Normalized Cross Correlation (NCC) of the modulus fields and the angle estimated by a SVD (Singular Value Decomposition)-based optimization model, respectively. With given thresholds on the modulus correlation and mean direction difference, the original field can be filtered with both the spatial structure of modulus distribution and the directions. The preliminary materials for constructing the methods are given in Sect. 2. In Sect. 3, we apply the method to extract the spatio-temporal evolution of the El Niño from global wind data. Finally, the conclusions are given in Sect. 4.

\section{Method}

\subsection{Multivector expression of vector fields in $C l_{2,0}$}

The most commonly used 2-D vector field expression is founded on Euclidean space with Cartesian coordinates. Their expressions are highly dependent on the coordinates. The Clifford algebra provides the coordinate-free expression and computation of vectors. With orthogonal Clifford algebra bases $\boldsymbol{e}_{1}$ and $\boldsymbol{e}_{2}$ in Clifford algebra space $C l_{2,0}$, any vector $\boldsymbol{v}$ in $C l_{2,0}$ space can be directly expressed as

$\boldsymbol{v}=v_{1} \boldsymbol{e}_{1}+v_{2} \boldsymbol{e}_{2}, \boldsymbol{v} \in R^{2} \subset C l_{2,0}$.

A discrete 2-D vector field $F: \Omega \subset R^{2} \rightarrow R^{2}$ can be expressed with a set of vectors in the following form:

$\boldsymbol{F}: \Omega \subset R^{2} \rightarrow R^{2}=\left\{\boldsymbol{x}_{i j}\right\}, i=1, \cdots, M, j=1, \cdots, N$

The geometric product can be defined for two vectors. For any given two vector $\boldsymbol{x}=x_{1} \boldsymbol{e}_{1}+y_{1} \boldsymbol{e}_{2}$ and $\boldsymbol{y}=x_{2} \boldsymbol{e}_{1}+y_{2} \boldsymbol{e}_{2}$, their geometric product can be defined as

$x y=x \cdot y+x \wedge y$.

In Eq. (3), the part constructed by inner product is a scalar. However, the part constructed by outer product is a bi-vector, which has $i_{2}=e_{1} e_{2}$ part. Multivector, one of the fundamental tools of Clifford algebra, integrates different dimensional subspace spanned by the basis elements in a single structure. The hybrid expression and unified computation of different dimensional subspace provide ultimate power of the Clifford algebra. In $\mathrm{Cl}_{2,0}$ space, there are only four elements, the scalar, $\boldsymbol{e}_{1}, \boldsymbol{e}_{2}$ and $\boldsymbol{e}_{12}=\boldsymbol{e}_{1} \boldsymbol{e}_{2}$. The scalar part, which is a constant, has a grade of 0 . Both the $\boldsymbol{e}_{1}$ and $\boldsymbol{e}_{2}$ parts are grade 1 vectors and the $\boldsymbol{e}_{12}$ part is a bi-vector of grade 2 . All the four parts can construct multivector functions in $\mathrm{Cl}_{2,0}$ space with a general form of $\boldsymbol{A}=a_{0}+a_{1} \boldsymbol{e}_{1}+a_{2} \boldsymbol{e}_{2}+a_{3} \boldsymbol{e}_{12}$. All the computations in $C l_{2,0}$ are closed in this general form. Different from the complex or tensorial approaches, the Clifford algebra approach encodes geometric objects of all dimensions (including all subspace dimensions) as algebraic objects and allows measurements of length, areas and volumes, and of dihedral angles across all dimensions (Hestenes and Sobcyk, 1984; De Bie et al., 2011). Clifford algebra allows coordinate free formulations and computation (Hestenes and Sobcyk, 1984).

\subsection{The Clifford convolution}

The definition of convolution is extended to vector field domain, and provides a template matching technology for vector field data (Ebling and Scheuermann, 2006). In discretized form, the convolution is computed directly by the geometric product. The center point value of the convolution result is the sum of the geometric product of all the corresponding values of the vector field and the vector template in the convolution window. Since the geometric product is composed by the inner and outer product, the convolution between two discrete 2-D vector fields $f(\boldsymbol{x})$ and $g(\boldsymbol{x})$ can be expressed as

$$
\begin{aligned}
& (f * g)(\boldsymbol{x})=\sum_{\boldsymbol{t}} f(\boldsymbol{t}) g(\boldsymbol{x}-\boldsymbol{t}) \\
& =\underbrace{\sum_{\boldsymbol{t}} f(\boldsymbol{t}) \cdot g(\boldsymbol{x}-\boldsymbol{t})}_{\text {The inner product part }}+\underbrace{\sum_{\boldsymbol{t}} f(\boldsymbol{t}) \wedge g(\boldsymbol{x}-\boldsymbol{t})}_{\text {The outer product part }} .
\end{aligned}
$$

In Eq. (4), the part constructed by the inner product is a scalar field, which indicates the angle differences between the original vector field and the template data in the sampling windows $\boldsymbol{t}$. However, the part constructed by the outer product is a bi-vector, which has $i_{2}$ part. In Ebling's template matching technology, the angle information is pre-processed separately, and only the magnitude fields are used in the final correlations computation (Ebling and Scheuermann, 2006).

Any 2-D vector can be linearized as a modulus field multiplied by a unit length vector. The assumption is that $f_{m}(\boldsymbol{x})=|f(\boldsymbol{x})|$ and $g_{m}(\boldsymbol{x})=|g(\boldsymbol{x})|$ are the modulus of geophysical vector field $f(\boldsymbol{x})$ and template field $g(\boldsymbol{x})$, and the normalized directional vector field $f_{\mathrm{d}}(\boldsymbol{x})=\left(f_{1}(\boldsymbol{x}), f_{2}(\boldsymbol{x})\right)$ and $g_{\mathrm{d}}(\boldsymbol{x})=\left(g_{1}(\boldsymbol{x}), g_{2}(\boldsymbol{x})\right)$ with unit length, which means $f_{1}(\boldsymbol{x})^{2}+f_{2}(\boldsymbol{x})^{2}=1$ and $\mathrm{g}_{1}(\boldsymbol{x})^{2}+g_{2}(\boldsymbol{x})^{2}=1$. Then there is a relation that $f_{1}(\boldsymbol{x}) g_{1}(\boldsymbol{x})+f_{2}(\boldsymbol{x}) g_{2}(\boldsymbol{x})=\cos \theta(\boldsymbol{x})$ and $f_{1}(\boldsymbol{x}) g_{2}(\boldsymbol{x})-f_{2}(\boldsymbol{x}) g_{1}(\boldsymbol{x})=\sin \theta(\boldsymbol{x})$, where $\theta(\boldsymbol{x})$ is a function which states the angular differences between $f(\boldsymbol{x})$ and $g(\boldsymbol{x})$ at each corresponding location $\boldsymbol{x}$. By separating the modulus field and the direction field, both the original vector field and the template field can be rewritten as $f(\boldsymbol{x})=f_{m}(\boldsymbol{t}) f_{\mathrm{d}}(\boldsymbol{t})=|f(\boldsymbol{x})|\left(f_{1}(\boldsymbol{x}) \boldsymbol{e}_{1}+f_{2}(\boldsymbol{x}) \boldsymbol{e}_{2}\right)$ and $g(\boldsymbol{x})=g_{m}(\boldsymbol{t}) g_{t}(\boldsymbol{t})=|g(\boldsymbol{x})|\left(g_{1}(\boldsymbol{x}) \boldsymbol{e}_{1}+g_{2}(\boldsymbol{x}) \boldsymbol{e}_{2}\right) . \quad$ With Eq. (4), we have

$$
\begin{aligned}
(f * g)(\boldsymbol{x}) & =\sum_{\boldsymbol{t}} f(\boldsymbol{t}) g(\boldsymbol{x}-\boldsymbol{t}) \\
& =\sum_{\boldsymbol{t}}\left(f_{m}(\boldsymbol{t}) f_{\mathrm{d}}(\boldsymbol{t})\right)\left(g_{m}(\boldsymbol{t}) g_{t}(\boldsymbol{t})\right) \\
& =|(f * g)(\boldsymbol{x})|\left(\cos \theta(\boldsymbol{x})+i_{2} \sin \theta(\boldsymbol{x})\right) \\
& =|(f * g)(\boldsymbol{x})| \boldsymbol{e}^{i_{2} \theta(\boldsymbol{x})}
\end{aligned}
$$

where $\theta(\boldsymbol{x})=\arctan \left(\frac{<(f * g)(\boldsymbol{x})>_{2}\left(-i_{2}\right)}{<(f * g)(\boldsymbol{x})>_{0}}\right)$. 
In Eq. (5), the convolution result of two vector field data $f(\boldsymbol{x})$ and $g(\boldsymbol{x})$ can be separated as two parts: the $|(f * g)(\boldsymbol{x})|$ part and the $\boldsymbol{e}^{i_{2} \theta(\boldsymbol{x})}$ part. The former part is a scalar field, which has a complicated structure and the latter part is a function which mostly indicates the angular information between the two vector field data $f(\boldsymbol{x})$ and $g(\boldsymbol{x})$ (Ebling and Scheuermann, 2005). Unfortunately, this angular information is not an exact expression of the angular difference between any arbitrary vector field. Any arbitrary linear vector field can be decomposed as a linear combination with two saddles, one source and one vortex field, but not all parts have the same meaning of the direction information. Although $\mathrm{Bu}-$ jack et al. (2012) defines the total rotation of any arbitrary two-dimensional vector fields and provides an estimation algorithm, it is not very easy to apply the algorithm into geophysical vector field data, especially when the amplitude of the vector field (i.e. the length of each vectors) is taken into consideration.

\subsection{The modified correlation product}

From Eq. (5), it is clear that the two parts are index of amplitude similarity and direction information respectively. Therefore, it will be possible to remodel the formula by replacing the computation of both the amplitude similarity and angular difference. For the scalar part, there are already lots of efficient algorithms that can be used, thus we can directly replace the scalar part with a numeric indicator that can express the interaction between the modulus to form a new signal. And for the angular part, the critical thing is how to estimate the overall difference between the vector fields. As proposed in Bujack et al. (2012), the total rotation can be seen as applying a rotor $R$ between the original and template direction field data. Therefore, an optimization method can be developed to estimate the least square mean angular difference between the two data. After that, we can then easily apply the threshold for both amplitude and angular-based filtering.

Traditional convolution between scalar fields only produce a value that indicates the relation between the two fields. But the result is not invariant with the change of amplitude and not robust to noise. The NCC coefficient overcomes these difficulties by normalizing the results, yielding a cosine-like correlation coefficient. For two scalar fields $f(\boldsymbol{x})$ and $g(\boldsymbol{x})$, the NCC index is defined similarly to the correlation coefficients as follows Lewis (1995):

$$
\operatorname{NCC}(\boldsymbol{x})=\frac{1}{n} \frac{\sum_{\boldsymbol{t}}[f(\boldsymbol{x}-\boldsymbol{t})-\bar{f}][g(\boldsymbol{t})-\bar{g}]}{\sqrt{\sum_{\boldsymbol{t}}[f(\boldsymbol{x}-\boldsymbol{t})-\bar{f}]^{2} \sum_{\boldsymbol{t}}[g(\boldsymbol{t})-\bar{g}]^{2}}},
$$

where $\bar{f}, \bar{g}$ are the means of $f(\boldsymbol{x})$ and $g(\boldsymbol{x})$ in the convolution window, respectively, and $n$ is the number of points in the window. The range of NCC is from 0 to 1 , thus the NCC field is a scalar field that measures the similarities of amplitudes of the two vector fields.
The direction differences between the two vector fields can be expressed by a rotor (Luo et al., 2012). For the two-direction fields $f_{\mathrm{d}}(\boldsymbol{x})=f_{1}(\boldsymbol{x}) \boldsymbol{e}_{1}+f_{2}(\boldsymbol{x}) \boldsymbol{e}_{2}$ and $g_{\mathrm{d}}(\boldsymbol{x})=$ $g_{1}(\boldsymbol{x}) \boldsymbol{e}_{1}+g_{2}(\boldsymbol{x}) \boldsymbol{e}_{2}$, the adaptive rotor estimation based on the SVD (Singular Value Decomposition) method can be applied. A versor equation can be defined with $f_{\mathrm{d}}(\boldsymbol{x})$ and $g_{\mathrm{d}}(\boldsymbol{x})$ with a rotor $R$ :

$f_{\mathrm{d}}(\boldsymbol{x})=R g_{\mathrm{d}}(\boldsymbol{x}) R^{-1}+\varepsilon(\boldsymbol{x})$.

So for $f_{\mathrm{d}}(\boldsymbol{x})=\boldsymbol{a}_{1}, \boldsymbol{a}_{2}, \cdots, \boldsymbol{a}_{i}$ and $g_{\mathrm{d}}(\boldsymbol{x})=\boldsymbol{b}_{1}, \boldsymbol{b}_{2}, \cdots, \boldsymbol{b}_{i}$, the objective function can be defined as

$$
\begin{aligned}
\min F(R) & =\sum_{i=1}^{k}\left(\boldsymbol{a}_{i}-R \boldsymbol{b}_{i} R^{-1}\right)^{2} \\
& =\sum_{i=1}^{k}\left(\boldsymbol{a}_{i}^{2}+\boldsymbol{b}_{i}^{2}-2<\boldsymbol{a}_{i} R \boldsymbol{b}_{i} R^{-1}>_{0}\right) .
\end{aligned}
$$

With a constraint condition of $R R^{-1}=1$, the most suitable rotor $R$ can be solved with a SVD model:

$$
R^{*}=\llbracket[\mathbf{V} \rrbracket \llbracket \mathbf{U} \rrbracket]^{T}
$$

where $R^{*}$ is the optional rotor estimated. [[U], $[\mathbf{V} \rrbracket$ are estimated by the SVD of $\llbracket f_{\mathrm{d}} \rrbracket$, and $\llbracket f_{\mathrm{d}} \rrbracket=\llbracket \mathbf{U} \rrbracket \llbracket \mathbf{S} \rrbracket \llbracket \mathbf{V} \rrbracket^{T}=$ $\sum_{i=1}^{k} \llbracket\left[\boldsymbol{b}_{i} \rrbracket \llbracket \boldsymbol{a}_{i} \rrbracket^{T}\right.$.

Rotor can be written as an exponential form of $R=\boldsymbol{e}^{-I \theta / 2}$ (Dorst et al., 2007), where $\theta$ and $I$ are the rotation angle and rotation plane. In the grade two fields space, the rotation plane is exactly the $\boldsymbol{e}_{12}$ plan, so the exponential equation can also be reformed as

$R=e^{-\frac{\theta}{2} i_{2}}=\cos \frac{\theta}{2}-i_{2} \sin \frac{\theta}{2}$,

then the $e^{i_{2} \theta(x)}$ part of Eq. (5) can be expressed as

$e^{i_{2} \theta(\boldsymbol{x})}=e^{-\frac{-2 \theta(\boldsymbol{x})}{2} i_{2}}=R(-2 \theta(\boldsymbol{x}))$.

Since the convolution of Eq. (5) is a multvector, by replacing the scalar part of Eq. (5) with the NCC coefficients and the estimated mean rotated angle $\theta^{*}(\boldsymbol{x})$, we can combine amplitude correlation and direction in a single equation. To make this equation meaningful and computable, we define the modified correlation product $\odot$ to express the similarities between $f(\boldsymbol{x})$ and $g(\boldsymbol{x})$ as follows:

$$
\begin{aligned}
& (f \odot g)(\boldsymbol{x}) \\
& =\underbrace{\operatorname{NCC}(\boldsymbol{x})}_{\text {Scalar Correlation }} \underbrace{\left[\cos \left(\theta^{*}(\boldsymbol{x})\right)+i_{2} \sin \left(\theta^{*}(\boldsymbol{x})\right)\right]}_{\text {Angular Vector Field }} \\
& =\operatorname{NCC}(\boldsymbol{x}) R\left(-2 \theta^{*}(\boldsymbol{x})\right) .
\end{aligned}
$$

In Eq. (12), the result of the modified correlation product suggests a structure similarity scalar field is multiplied by a multivector function of the angular relations. Thus, the modified correlation product can process both the magnitudes and angles synchronously in a signal equation. The physical meanings of both magnitudes and angular correlations are also maintained. 


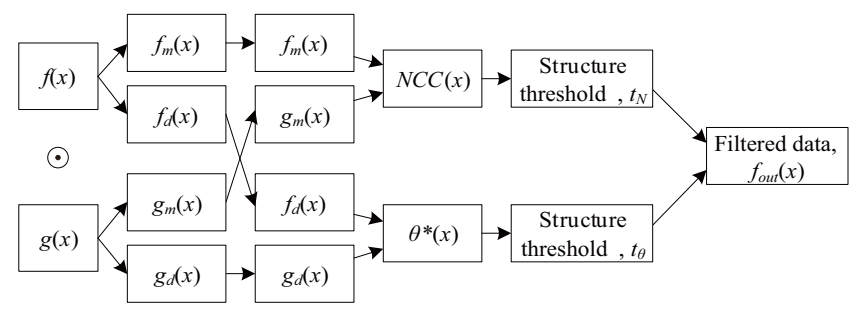

Fig. 1. The procedure of the template-based structure filtering.

\subsection{Template-based filtering of vector field in $\mathrm{Cl}_{2,0}$}

According to the discussion above, we can design the template-based filtering algorithm (Fig. 1). The original vector field data are first split into the modulus field and the unit length direction field. Since the modulus field is scalar field, we use the NCC computation algorithm proposed by Yoo and Han (2009). For the unit length direction field, the optimized angle difference field is first estimated by the SVD method. Then, we can apply a certain threshold on the NCC similarity $\operatorname{NCC}(\boldsymbol{x})$ and the angle $\theta^{*}(\boldsymbol{x})$ (e.g. a amplitude correlation more than 0.6 and the angular difference is less than $45^{\circ}$ ) to extract the features according to the relations between the original and template data.

\section{Experiments}

\subsection{Data}

Global wind filled and the El Niño Modoki event are studied. The $0.5^{\circ}$ Grided Global Average QuikSCAT Surface Wind Velocity Field data during May 2002 to March 2003 are used as the original data, and the Niño 3.4 region of the composite El Niño Modoki spatial pattern (Wang and Xin, 2013), which averages the NCEP-NCAR reanalysis wind velocity vector data during the seven El Niño Modoki events, are used as the template. Since the composed wind field data $\left(2.5^{\circ} \times 2.5^{\circ}\right)$ have a lower spatial resolution than the QuikSCAT wind velocity field data $\left(0.5^{\circ} \times 0.5^{\circ}\right)$, the composite data are first linearly interpolated in the $0.5^{\circ} \times 0.5^{\circ}$ grid.

\subsection{The directional filtering with parallel template}

Clifford convolution with a parallel template can extract the direction information of the vector field. A $11 \times 11$ parallel template $T(\boldsymbol{x})$ is generated with $T(\boldsymbol{x})=\sin [\theta(\boldsymbol{x})] \boldsymbol{e}_{1}+$ $\cos [\theta(\boldsymbol{x})] \boldsymbol{e}_{2}$ by assigning each point of a constant 2 -D vector. The north direction is set as $\theta(\boldsymbol{x})=0^{\circ}$. Therefore, the template is directly northward. The directional part of the modified correlation product indicates the direction of the angular difference from the north. We also compute the direction angle with the formula of $\theta(\boldsymbol{x})=\arctan (y / x)$ for comparison, where $x$ and $y$ are the longitudinal and latitudinal projection of the normalized direction vector in each point (Fig. 2).
The overall spatial patterns of the angle value calculated from both the convolution and the original data are very similar. This proves the correctness of our method. Furthermore, the highlighted area suggest our method will produce less singularities than the direct computation. The zonal distribution of the global wind (e.g. Western wind zone, Southeast trade wind zone etc.) can be clearly revealed, and from east to central Pacific $\left(10^{\circ} \mathrm{S} \sim 10^{\circ} \mathrm{N}, 120^{\circ} \mathrm{E} \sim 180^{\circ}\right)$, there are clearly boundaries of eastern and western wind. This area is also the birth place of El Niño event and a place that has drastic changes and large influence on global climate.

\subsection{The El Niño global impaction pattern extraction}

A time slice (September 2002) of the original wind field, the template data, the NCC coefficient and the directional classified angular coefficients are depicted in Fig. 3. The area with higher NCC coefficient may be strongly affected by this El Niño event. The high impact regions are located in the Equatorial Pacific between $30^{\circ} \mathrm{S} \sim 30^{\circ} \mathrm{N}$ and the east coastal regions of the main continents (Fig. 3c). The value of $\theta^{*}(\boldsymbol{x})$ reveals the angular relationship between the original data and the El Niño Modoki template, which indicate the directional structure change. For better expression, we classify the angular differences as eight directions. The direction classification of the filtered wind field is expressed in Fig. 3d.

The directional pattern of the wind field in September 2002 and the template clearly show distinct spatial distribution patterns in different areas. There are more eastward regions (in cold color) than westward regions (in warm color) in the whole global ocean. However, significant westward regions exist in the Pacific and Indian oceans, which suggests the closed linkage with ENSO. The most obvious westward region is located at East Pacific, east coast of South American, Africa, Asia, Australia and the west coast of Europe. The September 2002 is the forming stage of the El Niño event and the ENSO influences in these places are much stronger. The boundaries between the eastward and westward regions in central and east Pacific also have very similar structure to that of the weak El Niño composed by SST anomalies (Wang and Fiedler, 2006). Yu et al. (2012) suggests its SST anomaly center located near the coast of South America.

Since the spatial signal can be better separated from the frequency domain, testing the frequency characteristics of the modified correlation product is helpful. The 2-D Fourier spectrums of the original data, the template and the filtered field are computed and depicted in Fig. 4, which suggests that the modified correlation product can extract well and filter significant patterns and can also reveal the directional distribution. From Fig. 4, the spectrums also suggest clearly the filtering characteristics. In the original vector field, there are clearly periodical patterns, which have been filtered in the filtered field. The spectrum structures of the filtered field are more similar to the template field. For the directional patterns, there is no clear direction in original vector field. 

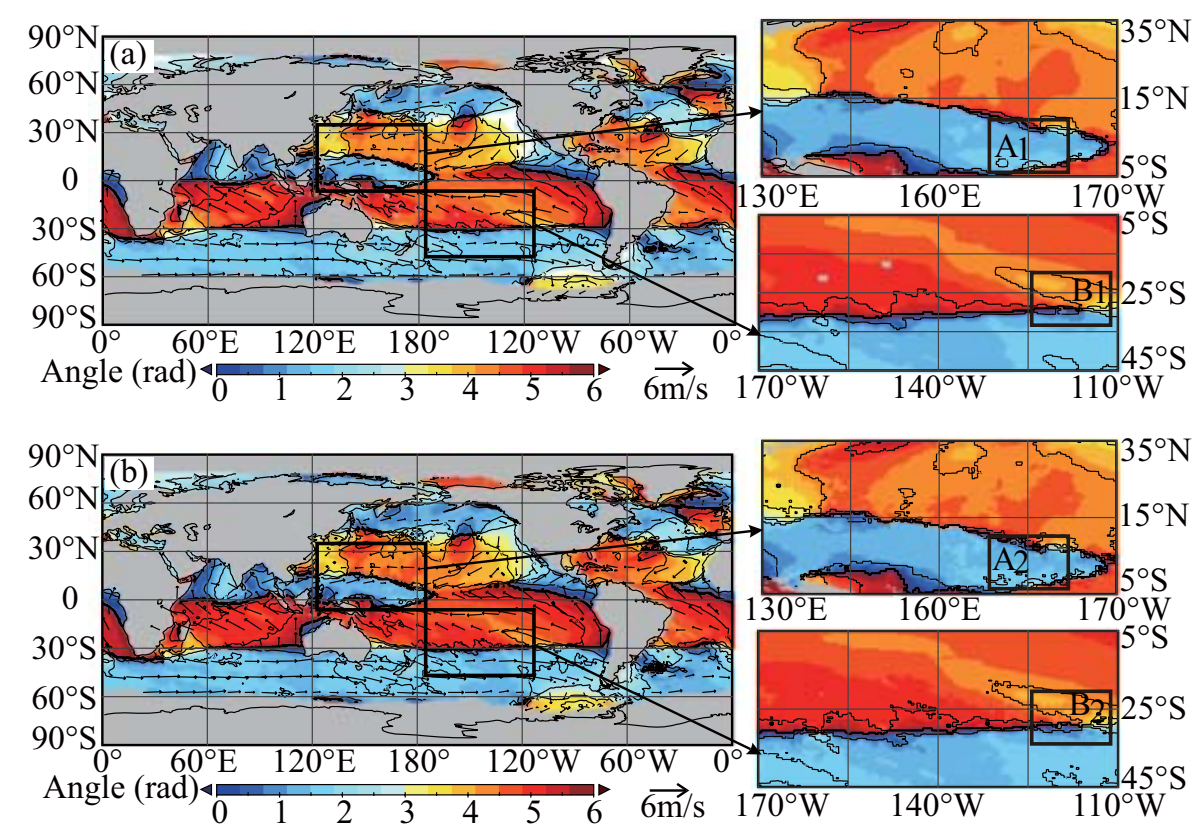

Fig. 2. The direction pattern comparison. (a) is the angle value calculated by optional rotor estimation, and (b) is calculated with the original vector data.
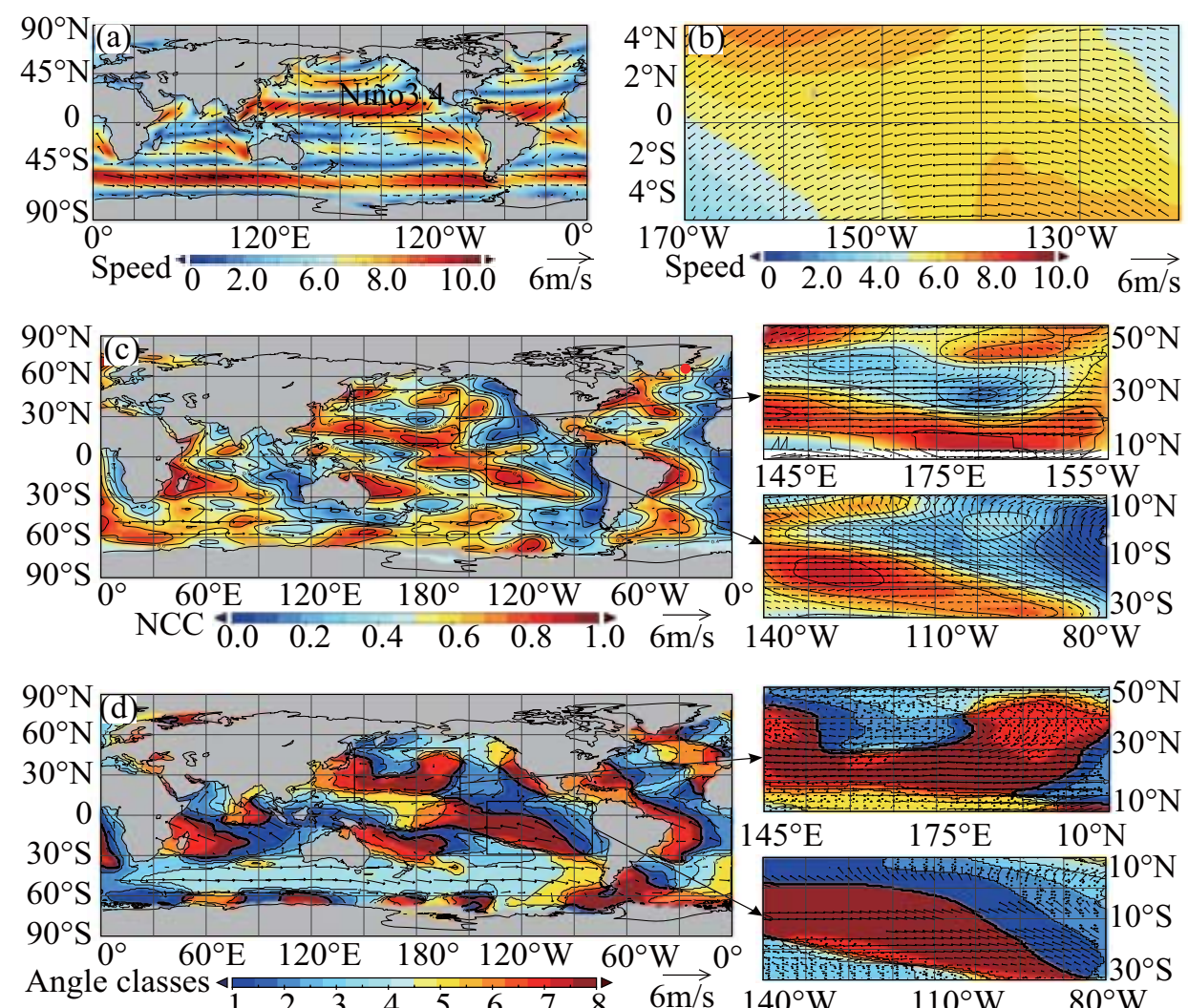

Fig. 3. The modified correlation product of September 2002. (a) is the original data, (b) is the template, and the NCC coefficient result and direction classification result can be seen in (c) and (d). 

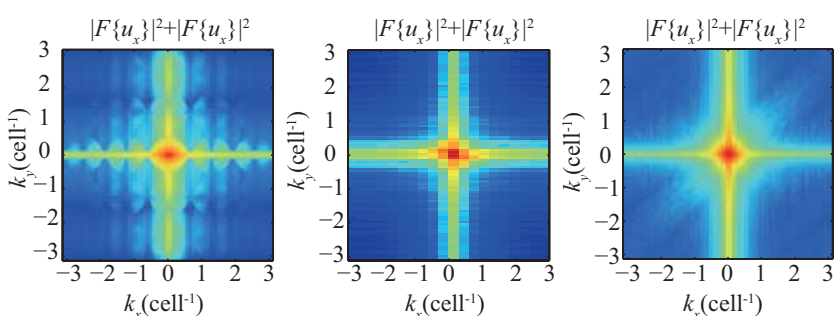

Fig. 4. The 2-D spectrum of original data (left), the template (middle) and the filtered field (right). The spectrum is the squared sum of the 2-D Fourier spectrum of the vectors projected in the two directions.

However, the direction distribution is clear and strengthened in the spectrum of the filtered field. These characteristics suggest the modified correlation product can be used for pattern extraction which integrated both the amplitude and directional part of the two vector fields.

\subsection{The evolution of high El Niño impact area}

Since both the NCC and the angular filtering can provide clear signals from vector field with template-based filtering, we can explore the tempo-spatial evolution characteristics of the 2002/03 El Niño event. The NCC coefficients changing with time indicate the amplitude similarity structure evolution, which may mainly suggest the strength of the El Niño event. And changing in the directional field classification indicates the directional effect evolution. In addition, the NCC threshold of NCC $>0.6$ is chosen to select the region highly related to or affected by the El Niño event. In the region of high NCC (Fig. 5a), the angular fields are divided within eight directional classes for better visualization (Fig. 5b). The distributions of the directions as well as the NCC coefficients are also expressed with the windrose plot, in which, the frequencies of the number of grid in each direction are counted and the associated NCC coefficients are expressed with the colorbar (Fig. 6).

The NCC coefficients suggest the structure change from May 2002 to March 2003. This El Niño event is strongly associated with active phases of the MJO (Madden-Julian Oscillation), an eastward propagating pattern affecting surface winds over the warm pool (Zhang, 2005). The characteristics of the MJO and its effects on surface wind can also be partially revealed in the data of NCC filtering. Several convective high NCC regions in the Pacific Ocean follow the eastward propagation of MJO. In May and June 2002, two of the highest NCC centers in the Pacific region appear in $\left(10^{\circ} \mathrm{N}, 170^{\circ} \mathrm{E}\right)$ and $\left(15^{\circ} \mathrm{S}, 130^{\circ} \mathrm{W}\right)$. But in July 2002 these two centers moved to $\left(15^{\circ} \mathrm{N}, 175^{\circ} \mathrm{E}\right)$ and $\left(25^{\circ} \mathrm{S}, 115^{\circ} \mathrm{W}\right)$. The whole high NCC regions in Pacific also totally show a clearly northeastward trend.

From August to October in 2002, the MJO related convection is growing weak. However, the original isolated peaks in the south ocean and the north Pacific are becoming connected, which suggests a global strengthening impact of the El Niño. Episodic forcing associated with the MJO helped to "kickstart" the 2002/03 El Niño (McPhaden, 2004), but then large-scale ocean-atmosphere feedbacks became the dominant forces that amplified and sustained the El Niño. In the maximum development of this event in OctoberDecember 2002, the area of the high NCC region also grows rapidly. In October 2002, in the Pacific region there are already two continuous zones that cross from $150^{\circ} \mathrm{E} \sim 90^{\circ} \mathrm{W}$. The slope of the El niño impact zone is even flatter. A strong similarity burst in the Pacific in November-December 2002 witnessed the amplification of the westerly wind bursts associated with the El Niño. And from January 2003, the area of the high NCC region declines. The termination of this El Niño event starts.

\subsection{The angular evolution of the El Niño event}

By classifying the direction distributions in the region of high NCC with the angular filtering results (Fig. 6), the highest NCC regions locate in Pacific, and the major direction pattern is northwestward. Compared to the shape and structure change of the high NCC region, the northwestward direction propagation is stable over time. In the boreal summer 2002, the MJO was dominated by a strong eastward component, probably driven by the abnormally high SSTs in the central Pacific (McPhaden, 2004). And in July 2002, the directional filtering result suggests the eastward becomes strong, which can be well compared with the observation results.

The center where the El Niño event developed is also a key problem for different patterns of the El Niño events. Existing literatures heavily argue the differences among the east $\mathrm{Pa}$ cific (EP) El Niño and central Pacific (CP) El Niño. In our angular direction classification, the central Pacific is located at the boundaries of the eastward and westward propagation. Other indicators also suggest the El Niño event was characterized by very high sea surface temperatures (SSTs) in the central Pacific (Kug et al., 2010). These further support that the central Pacific is the source of this El Niño event evolution. From the directional classification evolution we can also find the main propagation direction is almost stable over time (Fig. 7), although the original direction of the wind field changes continuously throughout time. In most time slices, the majority direction is the Northwest. The only exception is the July 2002, and there are opposite El Niño propagation directions on the east coast of China compared with that in North American. The influence of the heat and water transportation situation is also opposite (Cook et al., 2007).

\section{Conclusions}

The Clifford convolution is modified and applied to vector field analysis. By defining the modified correlation product 


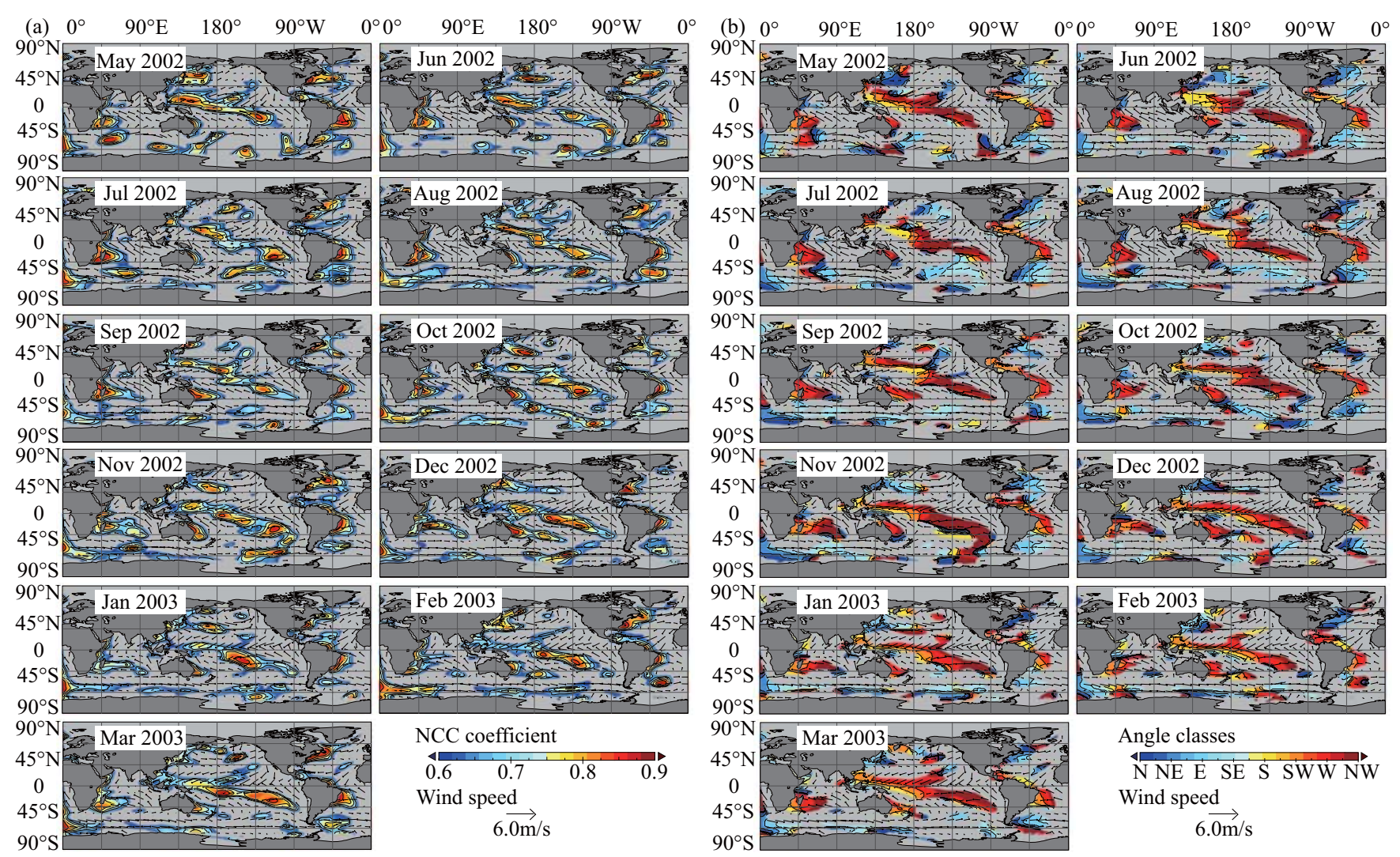

Fig. 5. The filtered results of modified correlation product with NCC $>0.6$ during May 2002 to March 2003. (a) is the NCC filtering results, and (b) is the direction classification filtering results.

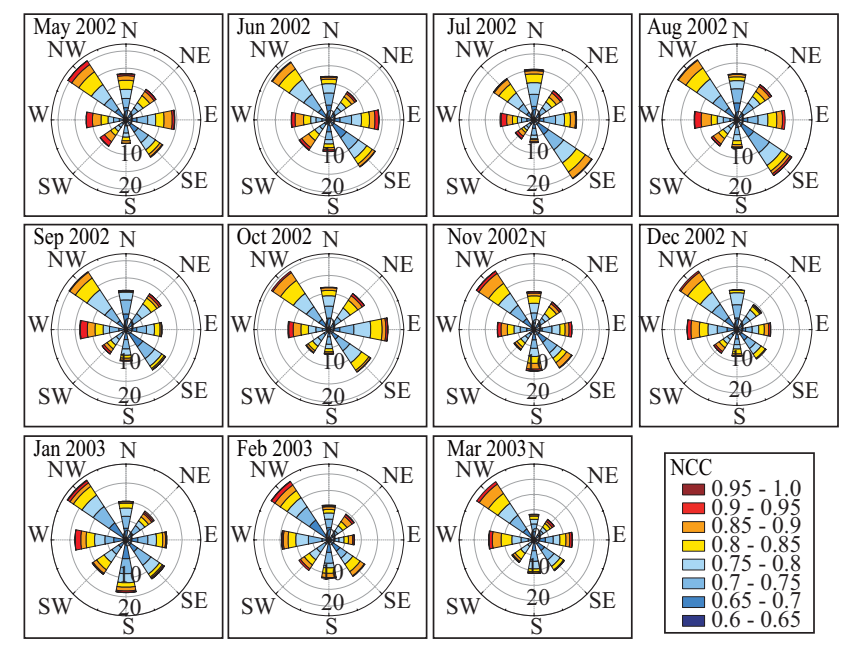

Fig. 6. The direction and NCC distribution of high $\mathrm{NCC}(>0.6)$ filtering results. The direction of the windrose indicates the evaluated direction calculated by the modified correlation product, and the velocity of the windrose suggests the magnitude of NCC. with the NCC and the SVD-based mean angle difference estimation method, both the amplitude and directional relations can be analyzed synchronously. The structure filtering algorithm can reveal both the modulus similarities as well as the angular distribution. The method can give smooth and continuous result, which may be helpful for vector data segmentation or classification. Future improvements, such as introducing more comprehensive correlation indexes as well as more stable and accurate amplitude correlation and angular estimation model, will greatly extend the application area.

With the El Niño template, the tempo-spatial evolution of the global El Niño impact and propagation can be revealed. It suggests the close linkage between the 2002/03 El Niño is first forced by the MJO event and then the large-scale oceanatmosphere feedbacks becomes dominant. Although the amplitude similarities vary in both temporal and spatial area, the directional propagation of El Niño is stable, especially for the areas with high amplitude similarities. This result may lead further studies on exploring the implied mechanisms about how the wind power and direction change interact with the El Niño evolution. 
Acknowledgements. This work was supported by the NSCF Project (Grant No. 41201377, 41231173) and NCET program (Grant No. NCET-12-0735).

Edited by: H. J. Fernando

Reviewed by: two anonymous referees

\section{References}

Brassington, G. B., Summons, N., and Lumpkin, R.: Observed and simulated Lagrangian and eddy characteristics of the East Australian Current and the Tasman Sea, Deep-Sea Res. Part II, 58, 559-573, 2011.

Bujack, R., Scheuermann, G., and Hitzer, E.: Detection of total rotations on 2D-vector fields with geometric correlation, in: AIP Conference Proceedings, edited by: Sivasundaram, S., vol. 1493, 190-199, AIP, available at: http://link.aip.org/link/APC/ 1493/190/1, 2012.

Cook, E. R., Seager, R., Cane, M. A., and Stahle, D. W.: North American drought: Reconstructions, causes, and consequences, Earth-Sci. Rev., 81, 93-134, 2007.

De Bie, H., De Schepper, N., and Sommen, F.: The class of CliffordFourier transforms, J. Fourier Analys. Appl., 17, 1198-1231, 2011.

Dorst, L., Fontijne, D., and Mann, S.: Geometric algebra for computer science: An object-oriented approach to geometry, Morgan Kaufmann, 2007.

Ebling, J. and Scheuermann, G.: Clifford Fourier transform on vector fields, IEEE Trans. Vis. Comput. Graphics, 11, 469-479, 2005.

Ebling, J. and Scheuermann, G.: Template matching on vector fields using Clifford algebra, in: Proceedings 17th IKM, edited by: Gürlebeck, K. and Könke, C., 1-25, 2006.

Hestenes, D. and Sobcyk, G.: Clifford algebra to geometric calculus, D. Reidel, Dordrecht, Holland, 1984

Kug, J.-S., Ahn, M.-S., Sung, M.-K., Yeh, S.-W., Min, H.-S., and Kim, Y.-H.: Statistical relationship between two types of El Niño events and climate variation over the Korean Peninsula, AsiaPac. J. Atmos. Sci., 46, 467-474, 2010.
Lewis, J.: Fast normalized cross-correlation, in: Proc. Vision Interface'95, 120-123, 1995.

Luo, W., Yuan, L. W., Yu, Z. Y., Yi, L., and Lü, G. N.: Multidimensional vector field convergence-divergence structure adaptive template matching method, Acta Electron. Sin., 40, 17291734, 2012.

McPhaden, M. J.: Evolution of the 2002/03 El Niño, Bull. Am. Meteorol. Soc., 85, 677-695, 2004.

Mendoza, C., Mancho, A. M., and Rio, M.-H.: The turnstile mechanism across the Kuroshio current: analysis of dynamics in altimeter velocity fields, Nonlin. Processes Geophys., 17, 103-111, doi:10.5194/npg-17-103-2010, 2010.

Paulus, C. and Mars, J. I.: New multicomponent filters for geophysical data processing, IEEE Trans. Geosci. Remote Sens., 44, 2260-2270, 2006.

Tafti, P. D. and Michael, U.: On regularized reconstruction of vector fields, IEEE Trans. Image Process., 20, 3163-3178, 2011.

Wang, C. and Fiedler, P.: ENSO variability and the eastern tropical Pacific: A review, Prog. Oceanogr., 69, 239-266, 2006.

Wang, C. and Xin, W.: EL Niño Modoki I and II classifying by different impacts on rainfall in Southern China and typhoon tracks, J. Climate, 26, 1322-1338, 2013.

Yoo, J. C. and Han, T. H.: Fast normalized cross-correlation, Circuits Syst. Signal Process., 28, 819-843, 2009.

Yu, J.-Y., Zou, Y., Kim, S. T., and Lee, T.: The changing impact of El Niño on US winter temperatures, Geophys. Res. Lett., 39, L15702, doi:10.1029/2012GL05248, 2012.

Yu, Z. Y., Yuan, L. W., Lü, G. N., Luo, W., and Xie, Z. R.: Coupling characteristics of zonal and meridional sea level change revealed by satellite altimetry data and their response to ENSO events, Chinese J. Geophys., 54, 1972-1982, 2011.

Yuan, L., Yu, Z., Luo, W., Yi, L., and Hu, Y.: Pattern forced geophysical vector field segmentation based on clifford FFT, Comput. Geosci., 60, 63-69, doi:10.1016/j.cageo.2013.05.007, 2013.

Zhang, C.: Madden-Julian oscillation, Rev. Geophys., 43, RG2003, doi:10.1029/2004RG000158, 2005. 